\title{
FRACTALS AND SELF-SIMILARITY IN ECONOMICS: THE CASE OF A STOCHASTIC TWO-SECTOR GROWTH MODEL
}

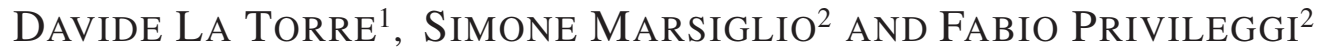 \\ ${ }^{1}$ Department of Economics, Business and Statistics, Università degli studi di Milano, via Conservatorio 7 , \\ I-20122 Milano, Italy; ${ }^{2}$ Department of Public Policy and Public Choice Polis, Università del Piemonte Orientale \\ Amedeo Avogadro, via Cavour 84, I-15121 Alessandria, Italy \\ e-mail: davide.latorre@unimi.it; simone.marsiglio@sp.unipmn.it; fabio.privileggi@sp.unipmn.it \\ (Accepted August 30, 2011)
}

\begin{abstract}
We study a stochastic, discrete-time, two-sector optimal growth model in which the production of the homogeneous consumption good uses a Cobb-Douglas technology, combining physical capital and an endogenously determined share of human capital. Education is intensive in human capital as in Lucas (1988), but the marginal returns of the share of human capital employed in education are decreasing, as suggested by Rebelo (1991). Assuming that the exogenous shocks are i.i.d. and affect both physical and human capital, we build specific configurations for the primitives of the model so that the optimal dynamics for the state variables can be converted, through an appropriate log-transformation, into an Iterated Function System converging to an invariant distribution supported on a generalized Sierpinski gasket.
\end{abstract}

Keywords: fractals, iterated function system, self-similarity, Sierpinski gasket, stochastic growth.

\section{INTRODUCTION}

Mandelbrot (1982) in his seminal work presented the first description of self-similar sets, namely sets that may be expressed as unions of rescaled copies of themselves. He called these sets fractals, because their (fractional) HausdorffBesicovitch dimensions exceeded their (integervalued) topological dimensions. The Cantor set, the von Koch snowflake curve and the Sierpinski gasket are some of the most famous examples of such sets. Hutchinson (1981) and, shortly thereafter, Barnsley and Demko (1985) and Barnsley (1989) showed how systems of contractive maps with associated probabilities, referred to as Iterated Function Systems (IFS), can be used to construct fractal, self-similar sets and measures supported on such sets. These sets and measures are attractive fixed points of fractal transform operators.

After these pioneering papers, applications of IFS theory in several fields have been widely developed, eventually landing, at the end of the last century, also into Economics. As a matter of fact, economists are intrinsically reluctant to accept the idea that economic dynamics may generate fractals. A first breakthrough has been introduced by Boldrin and Montrucchio (1986), who showed that complicated (chaotic) optimal dynamics can occur in deterministic concave intertemporal optimization models when the discount factor is small enough. This result opened a new chapter in mainstream Economics, starting a huge literature aimed at studying complexity and chaos in almost all economic fields. Prominent, but by no means exhaustive, ${ }^{1}$ references are Montrucchio (1994), Nishimura and Yano (1995), Brock and Hommes (1997) and, more recently, Gardini et al. (2009), who exploited the IFS framework to construct a deterministic OLG-model converging to a fractal attractor.

A decade later complex behavior started to be investigated in stochastic concave intertemporal optimization models as well. Montrucchio and Privileggi (1999) borrowed from the literature on fractal images generation (specifically, from the 'Collage Theorem' by Hutchinson, 1981; Barnsley, 1989; Vrscay, 1991) to show that standard stochastic concave optimal growth models may exhibit optimal trajectories which are random processes converging to singular invariant distributions supported on fractal sets regardless of the discount factor. Such economies have optimal dynamics defined by IFS with linear maps. Mitra et al. (2004) investigated a simple onesector growth model with two random shocks whose optimal path is defined by a linear IFS which, for some values of parameters, converges to a singular distribution supported on a Cantor set. They also characterized singularity versus absolute continuity of the invariant probability in terms of (almost) all

\footnotetext{
${ }^{1}$ For a recent and quite comprehensive survey on complex dynamics arising in non-competitive economies see Bischi et al. (2010) and the references listed there.
} 
parameters' values. Mitra and Privileggi $(2004,2006)$ further generalized that model and eventually (2009) provided an estimate of the Lipschitz constant for the (nonlinear) maps of the IFS defining the optimal policy in a class of stochastic one-sector optimal growth models in the Brock and Mirman (1972) tradition. This result yields sufficient conditions for the model to converge to a singular distribution supported on a generalized Cantor set directly in terms of the parameters' values.

In this paper we consider a neoclassic stochastic, discrete-time, two-sector growth model in which production of a unique homogeneous good depends on both physical and human capital through a Cobb-Douglas technology, while education requires only human capital, as suggested by Lucas (1988). However, we modify the Lucas (1988) framework by postulating that the marginal returns of the human capital employed in education are decreasing, thus embedding Rebelo (1991) assumption. Production in both sectors is multiplicatively affected by random i.i.d. shocks taking on a finite number of values. Our main contribution is to provide sufficient conditions on the parameters of the model - namely, on the exponents of the Cobb-Douglas production function and of the human capital production function, and on the values of random shocks - such that the IFS corresponding to the optimal policy function converges to a unique invariant distribution supported on a (generalized) Sierpinski gasket. Hence, this result can be seen as a further extension of the approach pursued by Mitra and Privileggi $(2004,2006,2009)$ for the onesector growth model to a multi-sector growth model under uncertainty.

In Section "Iterated function systems" the main results from the IFS theory are briefly recalled. In Section "The model", the model is stated and the optimal dynamics are explicitly computed. Section "Conjugate linear IFSP" contains the central contribution of this paper: a linear IFS conjugate to the true optimal dynamics is constructed and sufficient conditions for its attractor to be a Sierpinski gasket supporting the unique invariant distribution of the economy are provided directly in terms of parameters of the model. Finally, in Section "Examples of Sierpinski gasket-like attractors" a few examples of economies converging to differently shaped Sierpinski gaskets are described, while Section "Conclusions" reports some concluding remarks. All proofs are gathered in the Appendix.

\section{ITERATED FUNCTION SYSTEMS}

Iterated Function Systems allow to formalize the notion of self-similarity or scale invariance of some mathematical object. Hutchinson (1981) and Barnsley and Demko (1985) showed how systems of contractive maps with associated probabilities can be used to construct self-similar sets and measures. In the IFS literature, these are called IFS with probabilities (IFSP) and are based on the action of a contractive Markov operator on the complete metric space of all Borel probability measures endowed with the MongeKantorovich metric. Applications of these methods can be found in image compression, approximation theory, signal analysis, denoising, and density estimation (see, e.g., Freiberg et al., 2011; Kunze et al., 2007; Iacus and La Torre, 2005a,b; La Torre et al., 2006; La Torre and Mendivil, 2008, 2009; La Torre and Vrscay, 2009; La Torre et al., 2009; Mendivil and Vrscay, 2002a,b). In what follows, let $(X, d)$ be a complete metric space and $w=\left\{w_{1}, \ldots, w_{N}\right\}$ be a family of injective contraction maps $w_{i}: X \rightarrow X$, to be referred to as an $N$-map IFS. Let $c_{i} \in(0,1)$ denote the contraction factor of $w_{i}$ and define $c=\max _{i \in\{1, \ldots, N\}} c_{i}$. Note that $c \in(0,1)$. Associated with the IFS mappings $w_{1}, \ldots, w_{N}$ there is a set-valued mapping $\hat{w}: \mathscr{K}(X) \rightarrow \mathscr{K}(X)$ defined over the space $\mathscr{K}(X)$ of all non-empty compact sets in $X$ as

$$
\hat{w}(S)=\bigcup_{i=1}^{N} w_{i}(S), \quad S \in \mathscr{K}(X),
$$

where $w_{i}(S)=\left\{w_{i}(x): x \in S\right\}$ is the image of $S$ under $w_{i}$, for $i=1, \ldots, N$. A set $S_{w} \subset X$ is said to be an invariant set of $w$ if it is compact and it is invariant under Eq. 1, that is, it satisfies $\hat{w}\left(S_{w}\right)=S_{w}$. If in addition, the contractive mappings $w_{i}$ are assumed to be similitudes, i.e., if we assume that there exist numbers $c_{i} \in(0,1)$ such that

$d\left(w_{i}(x), w_{i}(y)\right)=c_{i} d(x, y), \quad x, y \in X, \quad i=1, \ldots, N$,

the invariant set $S_{w}$ is said to be self-similar. In $\mathscr{K}(X)$ it is possible to define the so-called Hausdorff distance $d_{H}$ between compact sets which reads as

$$
d_{H}(A, B)=\max \left\{\sup _{x \in A} \inf _{y \in B} d(x, y), \sup _{x \in B} \inf _{y \in A} d(x, y)\right\}
$$

and it can be proved that $\left(\mathscr{K}(X), d_{H}\right)$ is a complete metric space (see Hutchinson, 1981).

Theorem 1 (Hutchinson, 1981) $\hat{w}$ is a contraction mapping on the metric space $\left(\mathscr{K}(X), d_{H}\right)$, that is

$$
d_{H}(\hat{w}(A), \hat{w}(B)) \leq c d_{H}(A, B), \quad \forall A, B \in \mathscr{K}(X) .
$$

We have the following corollary from the Banach fixed point theorem. 
Corollary 1 There exists a unique compact set $A \in$ $\mathscr{K}(X)$, such that $\hat{w}(A)=A$, which is called the attractor of the IFS $w$. Moreover, for any $S \in \mathscr{K}(X)$, $d_{H}\left(\hat{w}^{n}(S), A\right) \rightarrow 0$ as $n \rightarrow \infty$.

The latter property provides a construction method of approximating a fractal. The equation $\hat{w}(A)=A$ obviously implies that $A$ is self-tiling, i.e., $A$ is the union of (distorted) copies of itself.

Let $\mathscr{M}(X)$ be the space of probability measures defined on the $\sigma$-algebra $\mathscr{B}(X)$ of Borel measurable subsets of $X$ and define for some $a \in X$ the set

$$
\mathscr{M}_{1}(X)=\left\{\mu \in \mathscr{M}(X): \int_{X} d(a, x) \mathrm{d} \mu(x)<\infty\right\} .
$$

Notice that the definition of $\mathscr{M}_{1}(X)$ does not depend on the choice of $a$ (if the integral is finite for a certain $a \in X$ then it is finite for all $a \in X)$. For $\mu, v \in \mathscr{M}_{1}(X)$, we define the Monge-Kantorovich distance as follows

$$
d_{M}(\mu, v)=\sup \left\{\int_{X} f d(\mu-v): f \in \mathscr{L} i p_{1}(X)\right\},
$$

where $\mathscr{L} i p_{1}$ is the set of all Lipschitz functions with Lipschitz constant equal to 1 . It can be proved that $\left(\mathscr{M}_{1}(X), d_{M}\right)$ is a complete metric space under the Monge-Kantorovich metric provided $X$ be a separable complete metric space. Furthermore, if $X$ is compact, then $\mathscr{M}(X)=\mathscr{M}_{1}(X)$ and both are compact metric spaces under the Monge-Kantorovich distance (see Barnsley et al., 2008).

Let $p=\left(p_{1}, p_{2}, \ldots, p_{N}\right), 0<p_{i}<1,1 \leq i \leq N$, be a partition of unity associated with the IFS mappings $w_{i}$, so that $\sum_{i=1}^{N} p_{i}=1$. Associated with this IFS with probabilities (IFSP) $(w, p)$ is the so-called Markov operator, $M: \mathscr{M}_{1}(X) \rightarrow \mathscr{M}_{1}(X)$, defined as

$$
(M \mu)(S)=\sum_{i=1}^{N} p_{i} \mu\left(w_{i}^{-1}(S)\right), \quad \forall S \in \mathscr{B}(X),
$$

where $w_{i}^{-1}(S)=\left\{y \in X: w_{i}(y) \in S\right\}$.

Theorem 2 (Barnsley et al., 2008) $M$ is a contraction mapping on the complete metric space $\left(\mathscr{M}_{1}(X), d_{M}\right)$, that is, for all $\mu, v \in \mathscr{M}_{1}(X)$

$$
d_{M}(M \mu, M v) \leq\left(\sum_{i} p_{i} c_{i}\right) d_{M}(\mu, v) .
$$

Corollary 2 There exists a unique probability measure $\bar{\mu} \in \mathscr{M}_{1}(X)$, called invariant measure of the $\operatorname{IFSP}(w, p)$, such that $M \bar{\mu}=\bar{\mu}$. Moreover, for any $\mu \in \mathscr{M}_{1}(X), d_{M}\left(M^{n} \mu, \bar{\mu}\right) \rightarrow 0$ as $n \rightarrow \infty$.
Note that for any $\mu$-integrable function $u: X \rightarrow \mathbb{R}$, it holds that

$$
\int_{X} u(x) \mathrm{d} \mu(x)=\sum_{i=1}^{N} p_{i} \int_{X} u\left[w_{i}(x)\right] \mathrm{d} \mu(x) .
$$

Let $C^{0}(X)$ denote the Banach space of continuous functions on $X$ endowed with the uniform metric $d_{\infty}$. Associated with the IFSP $(w, p)$ define the following operator $T: C^{0}(X) \rightarrow C^{0}(X)$ :

$$
T u=\sum_{i=1}^{N} p_{i}\left(u \circ w_{i}\right), \quad \forall u \in C^{0}(X) .
$$

For a given $v \in \mathscr{M}_{1}(X)$ define the linear functional $F_{v}: C^{0}(X) \rightarrow \mathbb{R}$ as

$$
F_{V}(u)=\langle u, v\rangle=\int_{X} u(x) \mathrm{d} v(x) .
$$

Then $\langle T f, v\rangle=\langle f, M v\rangle$, i.e., $T$ is the adjoint operator of $M$. The operator $T$ is a contraction on the complete metric space $\left(C^{0}(X), d_{\infty}\right)$ with contraction factor $p=$ $\max _{i \in\{1, \ldots, N\}} p_{i}<1$. Thus we have

$$
\int_{X} u(x) \mathrm{d} \mu(x)=\lim _{n \rightarrow+\infty} \int_{X} T^{n} f(x) \mathrm{d} \mu_{n}(x)
$$

where $\mu_{n}=M^{n} \lambda \rightarrow \mu$ in the Monge-Kantorovich distance and $\lambda$ is the Lebesgue measure on $X$.

It is worth mentioning the concept of $V$-variable fractals recently introduced by Barnsley et al. (2008) allowing for the description of new families of random fractals, which are intermediate between deterministic and random fractals, including recursive as well as homogeneous random fractals. More precisely, given a (not necessarily finite) family of IFSP's, such fractals are the result of random applications of the related set valued mappings and measure valued Markov operators. The parameter $V$ describes the degree of "variability" of the realizations. Roughly speaking, this means that at each construction step we have at most $V$ different fundamental shapes.

\section{THE MODEL}

We study an optimal growth model under uncertainty in which the social planner seeks to maximize the representative household's infinite discounted sum of instantaneous utility functions which are assumed to be logarithmic - subject to the laws of motion of physical, $k_{t}$, and human, $h_{t}$, capital. At each time $t$, the planner chooses consumption, $c_{t}$, and the share of human capital, $u_{t}$, to allocate into 
production of a unique homogeneous consumption good which uses a Cobb-Douglas technology that combines physical and human capital. Education is assumed to be intensive in human capital, as in Lucas (1988), but the marginal returns of the share of human capital employed in education are decreasing, in accordance with Rebelo (1991).

The final good and the education sectors are affected by exogenous perturbations, $z_{t}$ and $\eta_{t}$ respectively, which enter multiplicatively both production functions; they are independent and identically distributed, and take on finite values: $z \in\left\{q_{1}, q_{2}, 1\right\}$ and $\eta \in\{r, 1\}$, with $0<q_{1}<q_{2}<1$ and $0<r<1$. We assume that only three pairs of shock values can occur with positive probability, $(z, \eta) \in\left\{\left(q_{1}, r\right),\left(q_{2}, 1\right),(1,1)\right\}$, each with (constant) probability $p_{1}, p_{2}$ and $p_{3}$ respectively, where $p_{i} \in$ $(0,1), i=1,2,3$, and $\sum_{i=1}^{3} p_{i}=1$. Such three shock configurations may be interpreted as 1) a deep financial crisis typically having wide effects on the economy as a whole and thus involving both production and education sectors, ${ }^{2}$ corresponding to $(z, \eta)=\left(q_{1}, r\right)$, 2) a sudden surge in raw materials' (e.g., oil) prices affecting only the production sector but not education, corresponding to $(z, \eta)=\left(q_{2}, 1\right)$, and 3$)$ a scenario with no shocks in which the whole economy evolves along its full capacity, corresponding to $(z, \eta)=(1,1)$.

The social planner problem can thus be summarized as:

$$
\begin{gathered}
V\left(k_{0}, h_{0}, z_{0}, \eta_{0}\right)=\max _{\left\{c_{t}, u_{t}\right\}} \mathbb{E}_{0} \sum_{t=0}^{\infty} \beta^{t} \ln c_{t} \\
\text { s.t. }\left\{\begin{array}{l}
k_{t+1}=z_{t} k_{t}^{\alpha}\left(u_{t} h_{t}\right)^{1-\alpha}-c_{t} \\
h_{t+1}=\eta_{t}\left[\left(1-u_{t}\right) h_{t}\right]^{\phi}
\end{array}\right.
\end{gathered}
$$

where $k_{0}>0, h_{0}>0, z_{0} \in\left\{q_{1}, q_{2}, 1\right\}, \eta_{0} \in\{r, 1\}$ are given, $\mathbb{E}_{0}$ denotes expectation at time $t=0,0<\beta<1$ is the discount factor, $k_{t}$ and $h_{t}$ denote physical and human capital at time $t, 0<\alpha<1$ and $0<\phi<1$.

The Bellman equation associated to the problem defined in Eq. 2 reads as:

$$
\begin{aligned}
V\left(k_{t}, h_{t}, z_{t}, \eta_{t}\right) & =\max _{c_{t}, u_{t}}\left[\ln c_{t}\right. \\
& \left.+\beta \mathbb{E}_{t} V\left(k_{t+1}, h_{t+1}, z_{t+1}, \eta_{t+1}\right)\right] .
\end{aligned}
$$

Thanks to the log-Cobb-Douglas specification of the model, both the value function $V(\cdot, \cdot, \cdot, \cdot)$ and the optimal policy of the problem defined in Eq. 2 can be explicitly computed by applying the "guess and verify" method $^{3}$ to the Bellman equation (Eq. 4).

\section{Proposition 1}

1. The solution $V(k, h, z, \eta)$ of the Bellman equation in Eq. 4 is given by:

$$
\begin{aligned}
V(k, h, z, \eta)=\theta+\theta_{k} \ln k & +\theta_{h} \ln h \\
& +\theta_{z} \ln z+\theta_{\eta} \ln \eta
\end{aligned}
$$

where the constants $\theta_{k}, \theta_{h}, \theta_{z}$ and $\theta_{\eta}$ are defined as follows:

$$
\begin{aligned}
& \theta_{k}=\frac{\alpha}{1-\alpha \beta}, \quad \theta_{h}=\frac{1-\alpha}{(1-\alpha \beta)(1-\beta \phi)}, \\
& \theta_{z}=\frac{1}{1-\alpha \beta}, \quad \theta_{\eta}=\frac{(1-\alpha) \beta}{(1-\alpha \beta)(1-\beta \phi)},
\end{aligned}
$$

and the constant term $\theta$ is given by:

$$
\begin{aligned}
\theta=\frac{1}{1-\beta}[ & \ln (1-\alpha \beta)+\frac{\alpha \beta}{1-\alpha \beta} \ln (\alpha \beta) \\
& +\frac{1-\alpha}{1-\alpha \beta} \ln (1-\beta \phi) \\
+ & \frac{(1-\alpha) \beta \phi}{(1-\alpha \beta)(1-\beta \phi)} \ln (\beta \phi) \\
& +\frac{\beta}{(1-\alpha \beta)} \mathbb{E} \ln z \\
& \left.+\frac{(1-\alpha) \beta^{2}}{(1-\alpha \beta)(1-\beta \phi)} \mathbb{E} \ln \eta\right]
\end{aligned}
$$

2. The optimal policy rules for consumption and share of human capital allocated to physical production are respectively given by:

$$
\begin{aligned}
c_{t} & =(1-\alpha \beta)(1-\beta \phi)^{1-\alpha} z_{t} k_{t}^{\alpha} h_{t}^{1-\alpha} \\
u_{t} & =1-\beta \phi
\end{aligned}
$$

while physical and human capital follow the (optimal) dynamics defined by:

$$
\left\{\begin{array}{l}
k_{t+1}=\alpha \beta(1-\beta \phi)^{1-\alpha} z_{t} k_{t}^{\alpha} h_{t}^{1-\alpha} \\
h_{t+1}=(\beta \phi)^{\phi} \eta_{t} h_{t}^{\phi} .
\end{array}\right.
$$

The proof is reported in the Appendix.

An argument parallel to that described on pp. 273277 in Stokey and Lucas (1989) establishes that the function $V(k, h, z, \eta)$ defined in Eq. 5 is actually the value function of problem in Eq. 2.

\footnotetext{
${ }^{2}$ Consider, for example, the global financial crisis triggered in 2009: both the productive and education sector have been strongly damaged by the falling prices in the stock market.

${ }^{3}$ A similar approach has been pursued by Bethmann (2007) in a Lucas-Uzawa model of endogenous growth.
} 


\section{CONJUGATE LINEAR IFSP}

The optimal dynamics for the physical and human capital in Eq. 9 have the form of products of powers, suggesting that a logarithmic transformation of both variables $k_{t}$ and $h_{t}$ may yield an equivalent conjugate system which is linear in the transformed variables. Specifically, a suitable transformation of Eq. 9 may lead to a contractive IFSP converging to a unique invariant distribution supported on some fractal attractor in accordance with Corollaries 1 and 2 of Section "Iterated function systems". The following proposition shows that, for specific sets of values for parameters $\alpha, \phi, q_{1}, q_{2}$ an $r$, a linear system conjugate to Eq. 9 exists defining a IFSP that converges to an invariant distribution supported on a (generalized) Sierpinski gasket with vertices $(0,0),(1 / 2,1)$ and $(1,0)$.

Proposition 2 Assume that $\alpha \neq \phi$ and let

$$
r=\exp \left[\frac{\alpha-\phi}{1-\alpha}\left(2 \ln q_{2}-\ln q_{1}\right)\right] .
$$

Then the one-to-one logarithmic transformation $\left(k_{t}, h_{t}\right) \rightarrow\left(x_{t}, y_{t}\right)$ defined by:

$$
\left\{\begin{array}{l}
x_{t}=\rho_{a} \ln k_{t}+\rho_{b} \ln h_{t}+\rho_{c} \\
y_{t}=\rho_{d} \ln h_{t}+\rho_{e}
\end{array}\right.
$$

with

$$
\begin{aligned}
& \rho_{a}=-\frac{1-\alpha}{2 \ln q_{2}}, \quad \rho_{b}=\frac{(1-\alpha)^{2}}{2(\phi-\alpha) \ln q_{2}} \\
& \rho_{c}=1+\frac{1}{2 \ln q_{2}}\left\{\ln \left[\alpha \beta(1-\beta \phi)^{1-\alpha}\right]+\frac{1-\alpha}{\alpha-\phi} \ln \left[(\beta \phi)^{\phi}\right]\right\} \\
& \rho_{d}=\frac{(1-\alpha)(1-\phi)}{(\phi-\alpha)\left(2 \ln q_{2}-\ln q_{1}\right)} \\
& \rho_{e}=1+\frac{(1-\alpha) \ln \left[(\beta \phi)^{\phi}\right]}{(\alpha-\phi)\left(2 \ln q_{2}-\ln q_{1}\right)}
\end{aligned}
$$

defines a contractive linear IFSP which is equivalent to the nonlinear dynamics in Eq. 9 and is composed of the three maps $w_{1}, w_{2}, w_{3}: \mathbb{R}^{2} \rightarrow \mathbb{R}^{2}$, with probabilities $p_{1}, p_{2}, p_{3}$ respectively, given by:

$$
\left\{\begin{aligned}
\left(x_{t+1}, y_{t+1}\right) & =w_{1}\left(x_{t}, y_{t}\right)=\left(\alpha x_{t}, \phi y_{t}\right) \\
\left(x_{t+1}, y_{t+1}\right) & =w_{2}\left(x_{t}, y_{t}\right)=\left(\alpha x_{t}+(1-\alpha) / 2, \phi y_{t}+(1-\phi)\right) \\
\left(x_{t+1}, y_{t+1}\right) & =w_{2}\left(x_{t}, y_{t}\right)=\left(\alpha x_{t}+(1-\alpha), \phi y_{t}\right) .
\end{aligned}\right.
$$

The IFSP defined by Eq. 16 converges to an invariant distribution supported on a (generalized) Sierpinski gasket with vertices $(0,0),(1 / 2,1)$ and $(1,0)$.
The proof is reported in the Appendix.

Rewriting the IFSP in (16) as

$$
\left\{\begin{array}{l}
x_{t+1}=\alpha x_{t}+\gamma_{t} \\
y_{t+1}=\phi y_{t}+\vartheta_{t}
\end{array}\right.
$$

it is immediately seen that the three values $(0,0)$, $((1-\alpha) / 2,(1-\phi))$ and $((1-\alpha), 0)$ taken on by the (conjugate) random vector $\left(\gamma_{t}, \vartheta_{t}\right)$ correspond respectively to the three scenarios $\left(q_{1}, r\right),\left(q_{2}, 1\right)$ and $(1,1)$ for the original random values $(z, \eta)$ discussed in Section "The Model".

The mild restriction $\alpha \neq \phi$ required in Proposition 2 precludes the possibility of generating the standard Sierpinski gasket with vertices $(0,0),(1 / 2,1)$ and $(1,0)$ through Eq. 16, as its construction postulates that $\alpha=\phi=1 / 2$ must hold. In this sense, we say that the attractor of Eq. 16 is a generalized Sierpinski gasket. As it is clear from the proof, condition in Eq. 10 turns out to be the key restriction needed to construct the dynamics in Eq. 16 equivalent to those in Eq. 9.

\section{EXAMPLES OF SIERPINSKI GASKET-LIKE ATTRACTORS}

We consider four different parametrizations of the physical production and human capital production parameters, $\alpha$ and $\phi$. Note that any triple $0<q_{1}<$ $q_{2}<1$ and $0<r<1$ satisfying condition in Eq. 10 of Proposition 2 does the job; thus we do not set values for these parameters. Similarly, probabilities $p_{1}, p_{2}$ and $p_{3}$ can be any numbers between 0 and 1 summing up to 1 . In the first two scenarios, we tackle a framework very close to the benchmark case $\alpha=\phi=1 / 2$, corresponding to the standard Sierpinski gasket with vertices $(0,0),(1 / 2,1),(1,0)$ as the unique attractor of the IFSP in Eq. 16. As Proposition 2 requires $\alpha \neq \phi$, we set $\alpha=0.5$ and $\phi=0.49$. Fig. 1a shows the first 8 iterations ${ }^{4}$ of the map in Eq. 1 when the maps $w_{1}, w_{2}, w_{3}$ are given by Eq. 16 starting from the triangle of vertices $(0,0),(1 / 2,1),(1,0)$ as initial set $S_{0}$. While $\alpha=1 / 2$ implies that the two lower triangles of each prefractal ${ }^{5}$ have one vertex in common [e.g., point $(1 / 2,0)$ after one iteration], the assumption that $\phi<1 / 2$ implies that the top vertices of the two lower triangles are disjoint from the bottom vertices of the top triangle. Clearly, whenever $\alpha \geq 1 / 2$ and $\phi \geq 1 / 2$ with at least one strict inequality, all triangles in each prefractal overlap, as shown in Fig. 1b for $\alpha=0.5$ and $\phi=0.52$.

\footnotetext{
${ }^{4}$ The Maple 13 code for approximating the attractor of our economy under repeated iterations of the map in Eq. 1 is available from the authors upon request.

${ }^{5}$ The sets obtained after each iteration of the map in Eq. 1 are called prefractals.
} 

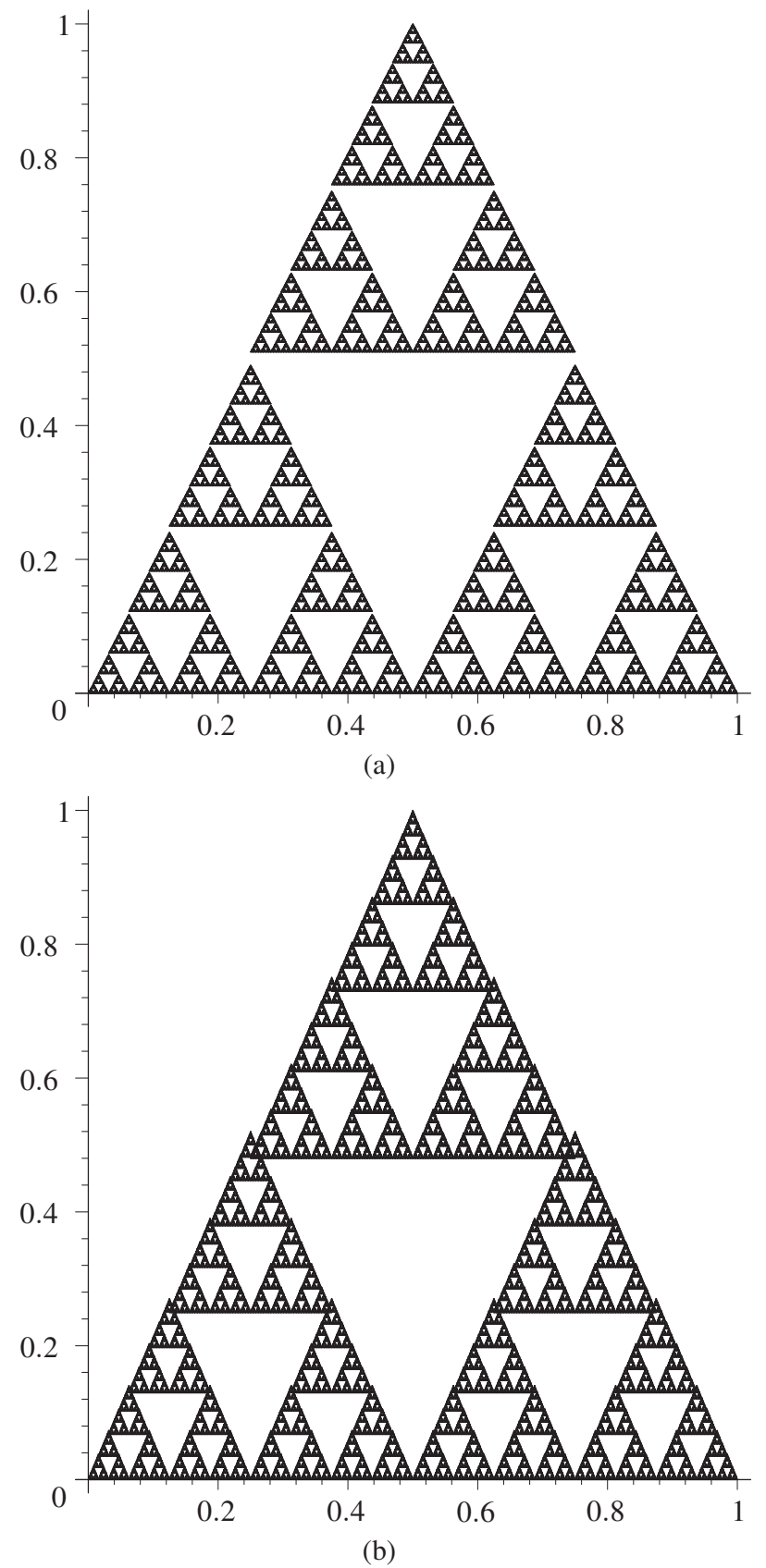

Fig. 1. First 8 iterations of the map in Eq. 1 for (a) $\alpha=1 / 2, \phi=49 / 100$, and (b) $\alpha=1 / 2, \phi=52 / 100$.

The last two cases consider a more realistic economy in which the capital share parameter is set to be $\alpha=0.333$. In the economic literature the capital share parameter in the output of the physical sector, $\alpha$, measuring its marginal returns on capital, has been traditionally considered the to be close to one third (Mankiw et al.,1992; Barro and Sala-i-Martin, 2004). A clear measure of the marginal returns of human capital in education has never been found in the empirical literature, since the human capital share in education is usually set to 1 in order to generate endogenous growth (Lucas, 1988). However, as argued by Rebelo (1991), we can reasonably assume that marginal returns of human capital are decreasing too. Probably, the most empirically relevant case is the one in which the education sector is relatively intensive in human capital, that is $\phi \leq 1-\alpha$ (Barro and Salai-Martin, 2004); therefore, in these two scenarios we assume a reasonable $\phi=0.5$ and a limiting case $\phi=$ $1-\alpha=0.667$. Figs. $2 \mathrm{a}$ and $2 \mathrm{~b}$ plot the first 7 iterations (which are enough in this case) of the map Eq. 1, again starting from the triangle of vertices $(0,0),(1 / 2,1)$ and $(1,0)$ as initial set $S_{0}$, for $\alpha=0.333, \phi=0.5$ and for $\alpha=0.333, \phi=0.667$ respectively.

\section{CONCLUSIONS}

In this paper we built a neoclassic, stochastic, discrete-time, two-sector optimal growth model in which the production of a homogeneous consumption good depends on physical and human capital. Our model exhibits two peculiar features: 1) the logCobb-Douglas structure of preferences plus production allows for a closed form solution of the Bellman equation, thus allowing for the explicit computation of the optimal dynamics of the state variables (Proposition 1), and 2) through a simple logtransformation of such dynamics we are able to show that for a sufficiently rich set of parameters' configurations this economy converges to an invariant distribution supported on a generalized Sierpinski gasket (Proposition 2). The only binding restriction is actually given by condition in Eq. 10 which relates the value $r$ of the shock affecting the education sector to the two values $q_{1}$ and $q_{2}$ of the shock affecting the production sector. However, we believe that our approach is sufficiently general as there is total freedom of choice on the values of two out of three exogenous shock parameters, leaving only the third dependent to the first two.

After investigating the (approximation of) the attractors of some economies in Figs. 1a, 1b, 2a and $2 b$, one may ask how the degree of overlapping among the prefractals may affect singularity properties of the invariant distribution. More precisely, it would be interesting to establish under what conditions on the model's parameters the invariant distribution turns out to be singular - or absolute continuous - with respect to Lebesgue measure. This exercise is left for future research. 


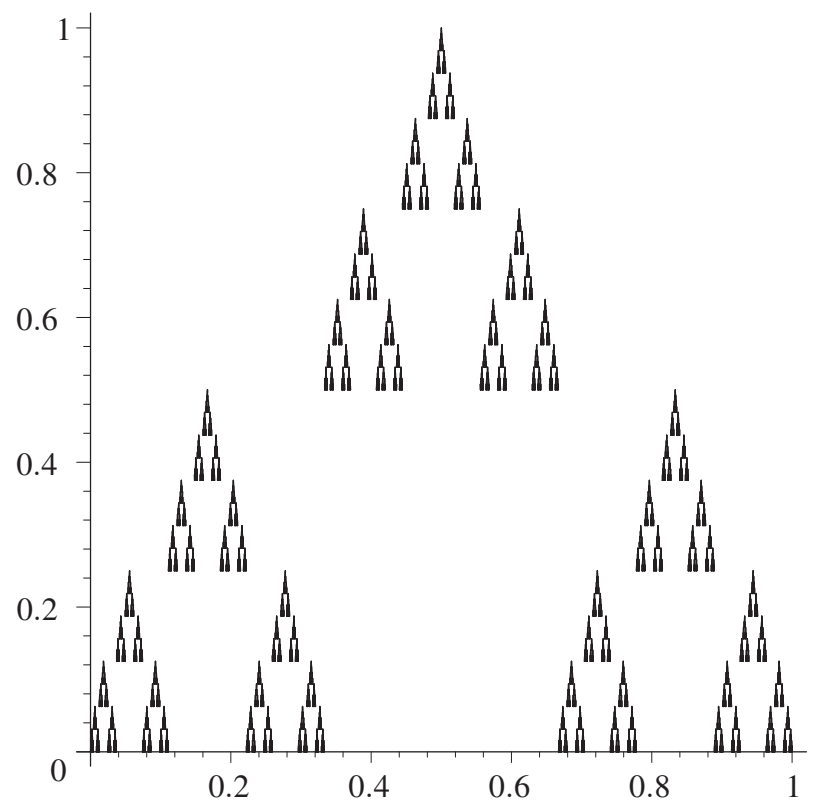

(a)

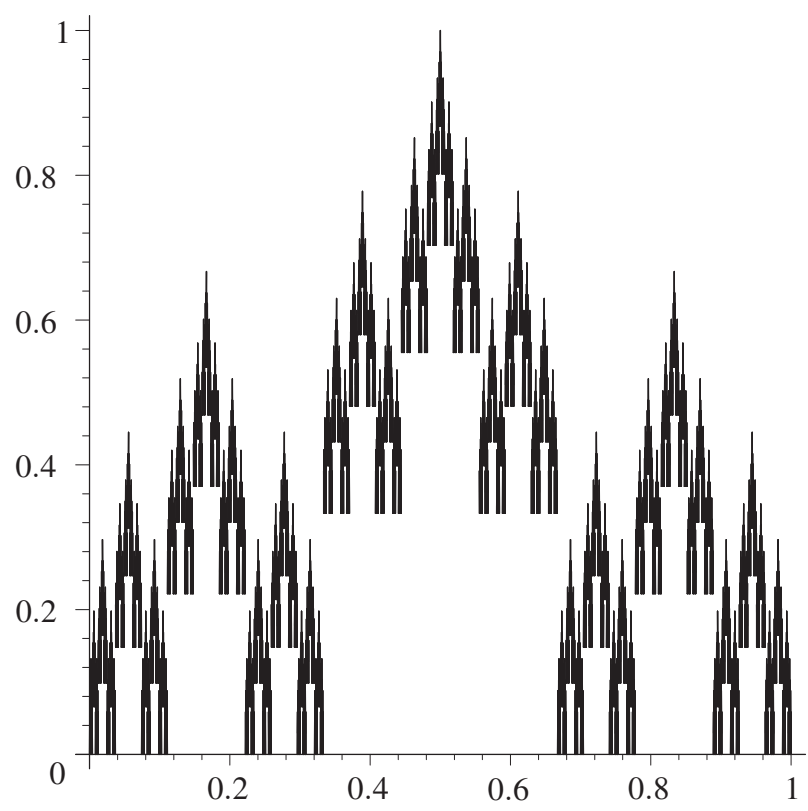

(b)

Fig. 2. First 7 iterations of the map in Eq. 1 for (a) $\alpha=1 / 3, \phi=1 / 2$, and (b) $\alpha=1 / 3, \phi=2 / 3$.

\section{APPENDIX}

Proof of Proposition 1. Assuming the form as in Eq. 5 for the value function and dropping the time subscript, the Bellman equation (Eq. 4) can be rewritten as:

$$
\begin{aligned}
\theta+\theta_{k} \ln k+\theta_{h} \ln h+\theta_{z} \ln z+\theta_{\eta} \ln \eta \\
=\max _{c, u}\left\{\ln c+\beta \theta+\beta \theta_{k} \ln \left[z k^{\alpha}(u h)^{1-\alpha}-c\right]\right. \\
+\beta \theta_{h} \ln \left[\eta(1-u)^{\phi} h^{\phi}\right] \\
\left.+\beta \theta_{z} \mathbb{E} \ln z+\beta \theta_{\eta} \mathbb{E} \ln \eta\right\} .
\end{aligned}
$$

FOC on the RHS with respect to $c$ and $u$ yield respectively:

$$
\begin{aligned}
\frac{1}{c} & =\frac{\beta \theta_{k}}{z k^{\alpha}(u h)^{1-\alpha}-c}, \\
\frac{\beta \phi \theta_{h}}{1-u} & =\frac{\beta \theta_{k}(1-\alpha) z k^{\alpha}(u h)^{-\alpha} h}{z k^{\alpha}(u h)^{1-\alpha}-c},
\end{aligned}
$$

while the envelope conditions read as:

$$
\begin{aligned}
\frac{\theta_{k}}{k} & =\frac{\alpha \beta \theta_{k} z k^{\alpha-1}(u h)^{1-\alpha}}{z k^{\alpha}(u h)^{1-\alpha}-c}, \\
\frac{\theta_{h}}{h} & =\frac{(1-\alpha) \beta \theta_{k} z k^{\alpha}(u h)^{-\alpha} u}{z k^{\alpha}(u h)^{1-\alpha}-c}+\frac{\beta \phi \theta_{h}}{h} .
\end{aligned}
$$

From Eq. 19 we get:

$$
c=\frac{1}{1+\beta \theta_{k}} z k^{\alpha}(u h)^{1-\alpha},
$$

which, when plugged into Eq. 21, after some algebra leads to:

$$
\theta_{k}=\frac{\alpha}{1-\alpha \beta}
$$

Using Eqs. 23 and 24 into Eq. 22, again after some algebra yields:

$$
\theta_{h}=\frac{1-\alpha}{(1-\alpha \beta)(1-\beta \phi)} .
$$

From Eqs. 20 and 22 we obtain $u=1-\beta \phi$, which is the optimal human capital share as in Eq. 8 while joining Eqs. 23 and 24 one immediately gets $c=$ $(1-\alpha \beta)(1-\beta \phi)^{1-\alpha} z k^{\alpha} h^{1-\alpha}$, which is the optimal consumption as in Eq. 7. The optimal dynamics (Eq. 9) are obtained by substituting Eqs. 7 and 8 into the dynamic constraints (Eq. 3).

Finally, in order to calculate the remaining constants $\theta, \theta_{z}$ and $\theta_{\eta}$ we substitute $\theta_{k}, \theta_{h}, c$ and $u$ as computed above into Eq. 18, so that the terms in $\ln k$ and $\ln h$ cancel out and we are left with:

$$
\begin{gathered}
\theta+\theta_{z} \ln z+\theta_{\eta} \ln \eta \\
=\ln (1-\alpha \beta)+\frac{1-\alpha}{1-\alpha \beta} \ln (1-\beta \phi) \\
+\beta \theta+\frac{\alpha \beta}{1-\alpha \beta} \ln (\alpha \beta)+\frac{(1-\alpha) \beta \phi}{(1-\alpha \beta)(1-\beta \phi)} \ln (\beta \phi) \\
+\frac{1}{1-\alpha \beta} \ln z+\frac{(1-\alpha) \beta}{(1-\alpha \beta)(1-\beta \phi)} \ln \eta+\beta \theta_{z} \mathbb{E} \ln z \\
+\beta \theta_{\eta} \mathbb{E} \ln \eta .
\end{gathered}
$$


For this equation to hold both the terms in $\ln z$ and $\ln \eta$ must vanish, which requires:

$$
\theta_{z}=\frac{1}{1-\alpha \beta} \quad \text { and } \quad \theta_{\eta}=\frac{(1-\alpha) \beta}{(1-\alpha \beta)(1-\beta \phi)},
$$

while $\theta$ turns out to be given by Eq. 6 .

Proof of Proposition 2. Using Eq. 11, Eq. 17 can be rewritten as:

$$
\left\{\begin{aligned}
\rho_{a} \ln k_{t+1}+\rho_{b} \ln h_{t+1}+\rho_{c}= & \alpha \rho_{a} \ln k_{t}+\alpha \rho_{b} \ln h_{t} \\
& +\alpha \rho_{c}+\gamma_{t} \\
\rho_{d} \ln h_{t+1}+\rho_{e}= & \phi \rho_{d} \ln h_{t}+\phi \rho_{e}+\vartheta_{t} .
\end{aligned}\right.
$$

Let us focus on the first equation in Eq. 25. Substituting $k_{t+1}$ and $h_{t+1}$ as in the first equation of Eq. 9, rearranging terms and after dropping the common terms $\alpha \rho_{a} \ln k_{t}$ such equation becomes:

$$
\begin{aligned}
& \rho_{a} \ln [\alpha \beta\left.(1-\beta \phi)^{1-\alpha}\right] \\
&+\rho_{b} \ln \left[(\beta \phi)^{\phi}\right]+(1-\alpha) \rho_{c} \\
&+\left[(1-\alpha) \rho_{a}+(\phi-\alpha) \rho_{b}\right] \ln h_{t} \\
&=\gamma_{t}-\rho_{a} \ln z_{t}-\rho_{b} \ln \eta_{t} .
\end{aligned}
$$

In order to let the constant $\rho_{c}$ be independent of $h_{t}$ in the equation above, we need that $(1-\alpha) \rho_{a}+$ $(\phi-\alpha) \rho_{b}=0$, so that the last term in the LHS cancels out and, under the assumption that $\alpha \neq \phi$, we have:

$$
\rho_{b}=\frac{1-\alpha}{\alpha-\phi} \rho_{a} .
$$

Using Eq. 27, Eq. 26 boils down to:

$$
\begin{gathered}
\left\{\ln \left[\alpha \beta(1-\beta \phi)^{1-\alpha}\right]+\frac{1-\alpha}{\alpha-\phi} \ln \left[(\beta \phi)^{\phi}\right]\right\} \rho_{a} \\
+(1-\alpha) \rho_{c} \\
=\gamma_{t}-\left[\ln z_{t}+\frac{1-\alpha}{\alpha-\phi} \ln \eta_{t}\right] \rho_{a} .
\end{gathered}
$$

As the LHS in Eq. 28 is constant, we can use the three values $\gamma_{t}=0, \gamma_{t}=(1-\alpha) / 2$ and $\gamma_{t}=$ $(1-\alpha)$, corresponding respectively to $\left(z_{t}, \eta_{t}\right)=$ $\left(q_{1}, r\right),\left(z_{t}, \eta_{t}\right)=\left(q_{2}, 1\right)$ and $\left(z_{t}, \eta_{t}\right)=(1,1)$ for the original shocks, and write:

$$
-\left[\ln q_{1}+\frac{1-\alpha}{\alpha-\phi} \ln r\right] \rho_{a}=\frac{1-\alpha}{2}-\rho_{a} \ln q_{2}=1-\alpha .
$$

From the second equation, using Eq. 27 we easily get $\rho_{a}$ and $\rho_{b}$ as in Eq. 12. Note, however, that the first equation on the left must hold as well, which, consistently with $\rho_{a}=-(1-\alpha) /\left(2 \ln q_{2}\right)$, is equivalent to condition in Eq. 10. As a matter of fact, condition in Eq. 10 is the key assumption to let Eq. 28 - or, equivalently, Eq. 26 - be independent of $h_{t}$. Substituting $\gamma_{t}=1-\alpha$ [corresponding to $\left(z_{t}, \eta_{t}\right)=$ $(1,1)]$ and $\rho_{a}$ as in Eq. 12 into Eq. 28 easily yields $\rho_{c}$ as in Eq. 13.

As far as the second equation in Eq. 25 is concerned, substituting $h_{t+1}$ as in the second equation of Eq. 9, rearranging terms and after dropping the common terms $\phi \rho_{d} \ln h_{t}$ such equation becomes:

$$
\rho_{d} \ln \left[(\beta \phi)^{\phi}\right]+(1-\phi) \rho_{e}=\vartheta_{t}-\rho_{d} \ln \eta_{t} .
$$

As the LHS is constant, we can use the two values $\vartheta_{t}=0$ and $\vartheta_{t}=(1-\phi)$, corresponding respectively to $\eta_{t}=r$ and $\eta_{t}=1$ for the original shocks on human capital, and write:

$$
-\rho_{d} \ln r=1-\phi,
$$

which immediately yields $\rho_{d}=-(1-\phi) / \ln r$, while $\rho_{e}=1+\ln \left[(\beta \phi)^{\phi}\right] / \ln r$ is obtained by plugging the expression of $\rho_{d}$ into Eq. 29. Finally, substituting $\ln r$ according to Eq. 10 yield $\rho_{d}$ and $\rho_{e}$ as in Eqs. 14 and 15.

As $0<\alpha<1$ and $0<\phi<1$, the IFSP in Eq. 16 - or, equivalently, Eq. 17 - is a contraction mapping; hence, Corollaries 1 and 2 apply and this is sufficient to show that the conjugate dynamics of system (Eq. 9) describing the optimal evolution of the state variable in our economy have a unique invariant distribution supported on a generalized Sierpinski gasket to which the economy converges in the long run.

\section{REFERENCES}

Barnsley MF (1989). Fractals everywhere. New York: Academic Press.

Barnsley MF, Demko S (1985). Iterated function systems and the global construction of fractals. Proc Roy Soc London Ser A 399:243-75.

Barnsley MF, Hutchinson J, Stenflo O (2008). V-variable fractals: fractals with partial self similarity. Adv Math 218:2051-88.

Barro RJ, Sala-i-Martin X (2004). Economic growth, 2nd Ed. Cambridge: MIT Press.

Bethmann D (2007). A closed-form solution of the UzawaLucas model of endogenous growth. J Econ 90:87-107.

Bischi GI, Chiarella C, Kopel M, Szidarovszky F (2010). Nonlinear oligopolies - stability and bifurcations. Berlin, Heidelberg: Springer-Verlag.

Boldrin M, Montrucchio L (1986). On the indeterminacy of capital accumulation paths. J Econ Theor 40:26-39.

Brock WA, Hommes CH (1997). A rational route to randomness. Econometrica 65: 1059-95. 
Brock WA, Mirman LJ (1972). Optimal economic growth and uncertainty: the discounted case. J Econ Theory 4:479-513.

Freiberg UR, La Torre D, Mendivil F (2011). Iterated function systems and stability of variational problems on self-similar objects. Nonlinear Anal Real 12:1123-9.

Gardini L, Hommes CH, Tramontana F, de Vilder R (2009). Forward and backward dynamics in implicitly defined overlapping generations models. J Econ Behav Organ 71:110-29.

Hutchinson J (1981). Fractals and self-similarity. Indiana Univ J Math 30:713-47.

Kunze H, La Torre D, Vrscay ER (2007). Contractive multifunctions, fixed point inclusions and iterated multifunction systems. J Math Anal Appl 330:159-73.

Iacus SM, La Torre D (2005a). A comparative simulation study on the IFS distribution function estimator. Nonlinear Anal Real 6:858-73.

Iacus SM, La Torre D (2005b). Approximating distribution functions by iterated function systems. J Appl Math Decis Sci 2005(1):33-46.

La Torre D, Mendivil F, Vrscay ER (2006). Iterated function systems on multifunctions. In: Aletti G, Burger M, Micheletti A, Morale D, eds. Math everywhere: deterministic and stochastic modelling in biomedicine, economics and industry. Berlin Heidelberg: SpringerVerlag. 125-34.

La Torre D, Mendivil F (2008). Iterated function systems on multifunctions and inverse problems. J Math Anal Appl 340:1469-79.

La Torre D, Mendivil F (2009). Union-additive multimeasures and self-similarity. Commun Math Anal 7:51-61.

La Torre D, Vrscay ER (2009). A generalized fractal transform for measure-valued images. Nonlinear Anal Theor 71:e1598-e1607.

La Torre D, Vrscay ER, Ebrahimi M, Barnsley MF (2009). Measure-valued images, associated fractal transforms, and the affine self-similarity of images. SIAM J Imaging Sci 2:470-507.

Lucas RE (1988). On the mechanics of economic development. J Monetary Econ 22:3-42.

Mandelbrot BB (1982). The fractal geometry of nature. San Francisco: Freeman.
Mankiw GN, Romer D, Weil DN (1992). A contribution to the empirics of economic growth. Q J Econ 107:407-37.

Mendivil F, Vrscay ER (2002a). Fractal vector measures and vector calculus on planar fractal domains. Chaos Soliton Fract 14:1239-54.

Mendivil F, Vrscay ER (2002b). Self-affine vector measures and vector calculus on fractals. In: Michael F. Barnsley MF, Saupe D, Vrscay ER, eds. Fractals in multimedia. Proc Meet Fractal Multimedia, 2001. IMA Vol Math Appl 132. New York: Springer-Verlag. 137-55.

Mitra T, Montrucchio L, Privileggi F (2004). The nature of the steady state in models of optimal growth under uncertainty. Econ Theor 23:39-72.

Mitra T, Privileggi F (2004). Cantor type invariant distributions in the theory of optimal growth under uncertainty. J Difference Equ Appl 10: 489-500.

Mitra T, Privileggi F (2006). Cantor type attractors in stochastic growth models. Chaos Soliton Fract 29:626-37.

Mitra T, Privileggi F (2009). On Lipschitz continuity of the Iterated Function System in a stochastic optimal growth model. J Math Econ 45:185-98.

Montrucchio L (1994). Dynamic complexity of optimal paths and discount factors for strongly concave problems. J Optim Theor Appl 80:385-406.

Montrucchio L, Privileggi F (1999). Fractal steady states in stochastic optimal control models. Ann Oper Res 88:183-97.

Nishimura K, Sorger G (1996). Optimal cycles and chaos: A survey. Stud Nonlinear Dynam Econometrics 1:11-28.

Nishimura K, Yano M (1995). Nonlinear dynamics and chaos in optimal growth: an example. Econometrica 63:981-1001.

Rebelo S (1991). Long-run policy analysis and long-run growth. J Polit Econ 99:500-21.

Stokey NL, Lucas RE (1989). Recursive methods in economic dynamics. Cambridge: Harvard University Press.

Vrscay ER (1991). Moment and collage methods for the inverse problem of fractal construction with iterated function systems. In: Peitgen HO, Henriques JM, Penedo LF, eds. Fractals in the fundamental and applied sciences. North-Holland. 443-461. 